\title{
Editorial commentary on the Indian Journal of Gastroenterology November-December 2020
}

\author{
Jimmy K. Limdi ${ }^{1,2,3}$ (D) \\ Published online: 18 January 2021 \\ (C) Indian Society of Gastroenterology 2021
}

\section{Outcome of hepatitis C-related liver transplantation in direct-acting antiviral era}

The effectiveness and safety of direct-acting antiviral agents (DAAs), the emergence of pan-genotypic drug regimens, and reduction in the cost of treatment have ushered in a new era for hepatitis $\mathrm{C}(\mathrm{HCV})$ treatment. However, the data supporting the efficacy of DAA treatment leading to patient-related outcomes are sparse [1]. Chaudhary and colleagues from Medanta the Medicity Hospital, Gurugram, India report a single centre experience of the changing severity and outcome profiles of HCVrelated liver disease at their institution before and after the availability of DAA's [2].

Among 410 patients undergoing liver transplant (LT) for HCV-related decompensated cirrhosis or hepatocellular carcinoma (HCC), HCV-related decompensated cirrhosis and/or HCC as an indication was reduced numerically in the period between 2015-2018 as compared to 2010-2014. Model for end-stage liver disease (MELD) scores were significantly lower in LT recipients with a better survival between 2015-2018 and significantly improved viral clearance (HCV negative status) before LT, compared to that between 2010-2014 [2]. Prospective and larger studies are needed to understand the impact of DAA's in $\mathrm{HCV}$ infection.

\section{Role of therapeutic plasma exchange in acute liver failure due to yellow phosphorus poisoning}

Yellow phosphorus is a protoplasmic toxin that can cause hepatocellular necrosis and hepatic failure, the only definitive

Jimmy K. Limdi

Jimmy.Limdi@nhs.net

1 Section of Inflammatory Bowel Diseases, Division of Gastroenterology, The Pennine Acute Hospitals NHS Trust, Manchester, UK treatment for it is liver transplantation (LT). Varghese and colleagues from the Institute of Liver Diseases and Transplantation, Gleneagles Global Health City, Chennai, India report their experience of using therapeutic plasma exchange (TPE) in 43 patients with acute liver failure (ALF) due to yellow phosphorus poisoning (YPP), 23 of whom did not meet LT criteria (group B) [3]. Although transaminases improved in both the groups, significant improvement in serum ammonia and $100 \%$ survival was noted in group B. Of 20 patients meeting LT criteria in group A, 4 underwent LT, 7 survived without LT and 9 died without LT. Statistically significant difference in survival (41.2 +/- 44.5 days in Group A and 90 days in group B) was noted, leading the authors to suggest that TP is effective in ALF due to YPP in patients not meeting criteria for LT. These findings are of interest, but more studies are needed.

\section{Single-balloon enteroscopy in management of small-bowel disorders}

Device-assisted endoscopy has enabled deep endoscopic small bowel access for hitherto unexplained gastrointestinal pathology but the literature on single balloon enteroscopy (SBE) is scarce [4]. Goenka et al. from Apollo Gleneagles Hospitals, Kolkata, India report a single-centre experience of investigation of suspected small bowel lesions by SBE in 158 patients [5]. The most common findings were ulcer (25\%), stricture $(4.44 \%)$ and both ulcers and strictures in $3.33 \%$ patients. SBE was normal in a third of patients, with a diagnostic yield in $66.11 \%$ and just under a third requiring therapeutic intervention. Complications included bleeding in 3 patients,

2 Manchester Academic Health Sciences, University of Manchester, Manchester, UK

3 Manchester Metropolitan University, Manchester, UK 
mild acute pancreatitis in 2 and jejunal perforation requiring surgical intervention in 1 patient.

Although SBE offers safe and effective deep direct endoscopic access to the small bowel, it is time consuming and requires specialist training. Further evaluation in larger prospective randomized trials as also comparative data with power-spiral enteroscopy, which may offer faster, deeper access and with a less invasive approach are needed [4].

\section{The efficacy and safety of colonoscopy in nonagenarians: A multicenter study}

Population aging is a global phenomenon [6]. In a thoughtprovoking study, Inagaki et al. from Nishijin Hospital, Kyoto, Japan, report on a multi-centre retrospective experience and outcomes of colonoscopy in nonagenarians, comparing this group to a non-elderly (50-64 years) and elderly (65-79 years) group of patients [7]. Among 16,969 cases, 125 nonagenarians received 154 colonoscopies. Using propensity score-matching of age, sex and colonoscopy indications, they analyzed the number of colorectal cancers (CRC) and treatment in each group. The rate of CRC among nonagenarians (27.2\%), was significantly higher than the non-elderly (7.2\%), and elderly $(8.8 \%)$ groups. Therapeutic interventions for CRC were performed in $73.5 \%$ of nonagenarian patients, with a colonoscopy-related complication rate of $1.3 \%$. Low sedation rates, meticulous selection of patients considering comorbidity and attention to bowel preparation are noteworthy. Whilst reassuring in themselves, these findings may not be generalizable, underpinning the need for careful consideration of patient, clinician and colonoscopy-related factors in the best interests of patients.

\section{Efficacies of first and second tumor necrosis factor inhibitors in refractory ulcerative colitis patients in real-world practice}

Anti-TNF therapies have transformed the care of inflammatory bowel disease, but these are immunogenic and associated with the loss of response [8]. Despite an increasing therapeutic armamentarium, it is advisable to optimize therapy within class when possible [9]. Marutani and colleagues report from Nagoya Memorial Hospital, Nagoya, Japan a retrospective experience of effectiveness of a second anti-TNF therapy after "failure" of first anti-TNF agent in patients with ulcerative colitis (UC) [10]. Among 123 UC patients, 21 switched within class. Reasons for switching included intolerance, loss of response and primary non-response to 1 st anti-TNF agent, respectively. Investigators noted that switching was more effective for patients who were intolerant to or had lost response to their first anti-TNF agent than primary non-responders. This underpins the need for astute clinical assessment using biomarkers, early recognition of primary and secondary loss of response. Although the authors did not specifically study the role of therapeutic drug monitoring, it can often help guide decisions.

\section{Splenic stiffness and platelet count to predict varices needing treatment in pediatric extrahepatic extrahepatic portal vein obstruction}

Despite extrahepatic portal venous hypertension (EHPVO) being an important cause of portal hypertension in adults and children, no guidelines or non-invasive parameters are currently available to identify varices needing endoscopic treatment (VNT) and surveillance. Platelet counts and liver stiffness measurement (LSM) are accepted criteria to predict VNT in cirrhosis. In EHPVO, LSM is near normal and splenic stiffness measurement (SSM) has been suggested as possibly more appropriate [11].

In a study of 107 children from Jawaharlal Institute of Postgraduate Medical Education and Research, Puducherry, India, evaluating the role of platelet counts and SSM to predict VNT (55 with EHPVO and 52 controls), 48/55 children with EHVPO had VNT [12]. Investigators noted no difference in platelet counts or SSM between children with EHPVO with VNT and those without [12]. The authors discuss nuances influencing their outcomes but suggest that until reliable non-invasive screening options for VNT are available, surveillance endoscopy may be necessary in children with EHPVO and splenomegaly.

\section{Development of integrated neonatal cholestasis card for early recognition and referral of neonatal cholestasis}

Neonatal cholestasis (NC), defined by the presence of jaundice with dark urine, constitutes $19 \%$ to $33 \%$ of hepatobiliary disorders among children in India. Delayed recognition is a significant source of morbidity and mortality from this disease [13]. Investigators from Taiwan reported an infant stool color card for use at the community level for the early diagnosis of biliary atresia (BA) [14]. Yachha and colleagues from Sanjay Gandhi Postgraduate Institute of Medical Sciences, Lucknow, India report a multi-centre study from India, evaluating the reliability of the stool card in an Indian population with the aim of developing an integrated $\mathrm{NC}$ card using urine and stool color identification [15].

Among 319 children (BA=58; non-BA=62 and 199 controls), without formal training, parents correctly identified dark yellow urine in all the children with $\mathrm{NC}$, and all the 50 
stool samples in children with BA with good correlation between parents and trainee doctors and paramedical staff members $(0.77$ and 0.64$)$, respectively. Formal training of pregnant mothers may be a significant step in the early identification of $\mathrm{NC}$ and mitigate against delays in detection and timely referral.

\section{Association between anti-acid therapies and advanced fibrosis in type 2 diabetics with biopsy-proven non-alcoholic fatty liver disease}

The commonality in risk factors and pathogenesis between non-alcoholic fatty liver disease (NAFLD) and type 2 diabetes (T2D) have led to NAFLD being considered as the hepatic manifestation of insulin resistance. Meanwhile proton-pump inhibitors (PPI's) have also been associated with adverse effects including enteric infections, chronic kidney disease, complications of cirrhosis and stroke [16]. Reduced acidity from PPI therapy, resultant dysbiosis, increased bacterial translocation and production of pro-inflammatory cytokines are implicated in hepatic fibrogenesis and its complications [16]. Singh et al. from Digestive Diseases and Surgery Institute, Cleveland Clinic, Ohio, USA assessed the association between $\mathrm{H}_{2}$-receptor antagonists $\left(\mathrm{H}_{2} \mathrm{RA}\right)$ and PPI's and advanced hepatic fibrosis in T2D [17]. Among 1008 T2D patients, $32 \%$ of whom had advanced fibrosis, $34 \%$ on $\mathrm{H}_{2}$ RA's and $60.6 \%$ on PPI's (median 3.6 years), PPIs (but not $\mathrm{H}_{2} \mathrm{RAs}$ ) were associated with a higher risk (OR 1.4) of advanced hepatic fibrosis. The study further highlights the need for judicious use of PPI. Larger prospective and mechanistic studies are needed to understand this association.

\section{Spectrum of mutations in genes associated with familial colorectal cancer syndrome (MLH1, MSH2, PMS2, MSH6, and APC): A not so common hereditary cancer syndrome in Indian population}

Although it is well recognized that the incidence of colorectal cancer (CRC) in India is less than in Western countries, data on inherited CRC syndromes from India are scarce. Bhai et al. from Sir Ganga Ram Hospital, New Delhi, India report their experience of proband and genetic testing in 36 families suspected to have a CRC syndrome [18]. Germline pathogenic variants were identified in the associated genes in 18 of 19 affected probands tested with 13 HNPCC, 5 with polyposis and 1 with Cowden syndrome. The implications of undetected familial CRC syndromes are serious. Along with greater awareness and index of suspicion, wider availability of genetic testing and counseling are both necessary as are further studies to assess the true prevalence of highly penetrant, albeit less prevalent familial CRC in the Indian population.

\section{Prevalence of elevated alanine aminotransferase levels in adult participants from a community-based study from northern part of India}

Alanine transaminase (ALT) is a cytosolic enzyme that leaks into circulation following hepatocytic injury from a wide range of etiologies [19]. Aggarwal and colleagues, from All India Institute of Medical Sciences, New Delhi, India report the prevalence of an elevated ALT from a retrospective analysis of a previous community-based prospective study (studying the prevalence of celiac disease) from a total of 33 villages in 27 urban wards in India [20].

Among 6083 patients tested, ALT was elevated above upper limit of normal (ULN) in $20.5 \%$ and $\geq 1.5 \mathrm{X}$ ULN in $5.4 \%$ of participants with higher prevalence among men. The authors also noted a strong correlation between elevated ALT and higher body mass index. Although investigation of the etiology of elevated ALT was not within the remit of this study, the high point prevalence in asymptomatic individuals indicates a public health issue given the potential for ongoing hepatocellular injury progressing to chronic liver disease.

\section{IgG4-related sclerosing cholangitis- A great mimicker}

IgG4-related sclerosing cholangitis, also known as autoimmune cholangiopathy is a chronic inflammatory disease of the biliary system that often occurs in association with other manifestations of IgG4-related systemic disease but may also occur in isolation [21].

It often mimics malignancy of the biliary tract or even others sclerosing cholangiopathies. A thorough knowledge of IgG4-related sclerosing cholangitis is essential to suspect the disease, perform appropriate investigations and administer suitable treatment. In this issue of the Indian Journal of Gastroenterology, Nayak and colleagues from All India Institute of Medical Sciences, Bhubaneswar, India describe an adolescent and adult cases of IgG4-related sclerosing cholangitis and their experience of assessment and treatment with budesonide or followed by corticosteroid therapy without a histological diagnosis [22]. The challenges and limitations of their approach are described in the case series presented.

\section{Compliance with ethical standards}

Conflict of interest JKL declares that he has no conflict of interest. 
Disclaimer The author is solely responsible for the data and the contents of the paper. In no way, the Honorary Editor-in-Chief, Editorial Board Members, the Indian Society of Gastroenterology, or the printer/ publishers are responsible for the results/findings and content of this article.

\section{References}

1. Abraham GM, Obley AJ, Humphrey LL, Qaseem A. World health organization guidelines on treatment of hepatitis $\mathrm{C}$ virus infection: best practice advice from the American College of Physicians. Ann Intern Med. 2020. https://doi.org/10.7326/M19-3860.

2. Choudhary NS, Saraf N, Saigal S, et al. Outcome of hepatitis Crelated liver transplantation in direct-acting antiviral era. Indian $\mathrm{J}$ Gastroenterol. 2020;39. https://doi.org/10.1007/s12664-02001105-z.

3. Varghese J, Joshi V, Bollipalli MK, et al. Role of therapeutic plasma exchange in acute liver failure due to yellowphosphorus poisoning. Indian J Gastroenterol. 2020;39. https://doi.org/10.1007/ s12664-020-01095-y.

4. Schneider M, Hollerich J, Beyna T. Device-assisted enteroscopy: a review of available techniques and upcoming new technologies. World J Gastroenterol. 2019;25:3538-45.

5. Goenka MK, Afzalpurkar S, Rai VK, et al. Single-balloon enteroscopy in management of small-bowel disorders. Indian $\mathrm{J}$ Gastroenterol. 2020;39. https://doi.org/10.1007/s12664-02001088-x.

6. WHO. https://www.who.int/news-room/fact-sheets/detail/ageingand-health.

7. Inagaki Y, Yoshida N, Hasegawa D, et al. The efficacy and safety of colonoscopy in nonagenarians: a multicenter study. Indian J Gastroenterol. 2020;39. https://doi.org/10.1007/s12664-02001066-3.

8. Alkhatry M, Al-Rifai A, Annese V, et al. First United Arab Emirates consensus on diagnosis and management of inflammatory bowel diseases: a 2020 Delphi consensus. World J Gastroenterol. 2020;26:6710-69.

9. Patel RN, Nigam GB, Jatale RG, et al. An Indian national survey of therapeutic drug monitoring with anti-tumor necrosis (TNF) medications in inflammatory bowel disease. Indian J Gastroenterol. 2020;39:176-85.

10. Marutani Y, Mizoshita T, Sugiyama T, et al. Efficacies of first and second tumor necrosis factor inhibitors in refractory ulcerative colitis patients in real-world practice. Indian J Gastroenterol. 2020;39. https://doi.org/10.1007/s12664-020-01092-1.
11. Madhusudhan KS, Sharma R, Kilambi R, et al. 2D shear wave Elastography of liver in patients with primary Extrahepatic portal vein obstruction. J Clin Exp Hepatol. 2017;7:23-7.

12. Vadlapudi SS, Jagadisan B, Ananthkrishnan R, Narayanaswamy S. Splenic stiffness and platelet count to predict varices needing treatment in pediatric extra-hepatic extrahepatic portal vein obstruction Indian J Gastroenterol 2020;39. https://doi.org/10.1007/s12664020-01099-8.

13. Bhatia V, Bavdekar A, Matthai J, et al. Management of neonatal cholestasis: consensus statement of the pediatric gastroenterology chapter of Indian academy of pediatrics. Indian Pediatr. 2014;51: 203-10.

14. Chen SM, Chang MH, Du JC, et al. Screening for biliary atresia by infant stool color card in Taiwan. Pediatrics. 2006;117:1147-54.

15. Yachha SK, Das MC, Kumar P, et al. Development of integrated neonatal cholestasis card for early recognition and referral of neonatal cholestasis. Indian J Gastroenterol. 2020;39. https://doi.org/ 10.1007/s12664-020-01094-Z.

16. Perry IE, Sonu I, Scarpignato C, et al. Potential proton pump inhibitor-related adverse effects. Ann N Y Acad Sci. 2020. https:// doi.org/10.1111/nyas.14428.

17. Singh A, Garg R, Lan N, et al. Association between anti-acid therapies and advanced fibrosis in type 2 diabetics with biopsy-proven non-alcoholic fatty liver disease. Indian J Gastroenterol. 2020;39. https://doi.org/10.1007/s12664-020-01087-y.

18. Bhai P, Kulshrestha S, Puri RD, Bijarnia Mahay S, Saxena R, Verma IC. Spectrum of mutations in genes associated with familial colorectal cancer syndrome (MLH1, MSH2, PMS2, MSH6, and APC): A not so common hereditary cancer syndrome in Indian population Indian J Gastroenterol. 2020;39. https://doi.org/10. 1007/s12664-020-01096-x.

19. Agrawal S, Dhiman RK, Limdi JK. Evaluation of abnormal liver function tests. Postgrad Med J. 2016;92:223-34.

20. Aggarwal N, Singh A, Agarwal A, et al. Prevalence of elevated alanine aminotransferase levels in adult participants from a community-based study from northern part of India. Indian J Gastroenterol. 2020;39. https://doi.org/10.1007/s12664-02001091-2.

21. Madhusudhan KS, Das P, Gunjan D, et al. IgG4-related Sclerosing cholangitis: a clinical and imaging review. AJR Am J Roentgenol. 2019;213:1221-31.

22. Nayak HK, Bhat SJ, Panigrahi MK, Chouhan I, Kumar C, Samal SC. IgG4-related sclerosing cholangitis- a great mimicker. Indian J Gastroenterol 2020;39. https://doi.org/10.1007/s12664-020-01102-2.

Publisher's note Springer Nature remains neutral with regard to jurisdictional claims in published maps and institutional affiliations. 